\title{
Managing magnetic resonance imaging machines: support tools for scheduling and planning
}

Adam P. Carpenter

Lawrence Leemis

William \& Mary, Imleem@wm.edu

Alan .S. Papir

David J. Phillips

Grace S. Phillips

Follow this and additional works at: https://scholarworks.wm.edu/aspubs

Part of the Mathematics Commons

\section{Recommended Citation}

Carpenter, Adam P.; Leemis, Lawrence; Papir, Alan .S.; Phillips, David J.; and Phillips, Grace S., Managing magnetic resonance imaging machines: support tools for scheduling and planning (2011). Health Care Management Science, 14, 158-173.

https://doi.org/10.1007/s10729-011-9153-z

This Article is brought to you for free and open access by the Arts and Sciences at W\&M ScholarWorks. It has been accepted for inclusion in Arts \& Sciences Articles by an authorized administrator of W\&M ScholarWorks. For more information, please contact scholarworks@wm.edu. 


\title{
Managing Magnetic Resonance Imaging machines: support tools for scheduling and planning
}

\author{
A. Carpenter, L. M. Leemis ${ }^{\dagger}$ A. S. Papirł D. J. Phillips $\$$ and G. S. Phillips
}

\begin{abstract}
We devise models and algorithms to estimate the impact of current and future patient demand for examinations on Magnetic Resonance Imaging (MRI) machines at a hospital radiology department. Our work helps improve scheduling decisions as well as support MRI machine purchasing decisions. Of particular novelty is our use of scheduling algorithms to compute the competing objectives of maximizing examination throughput and patient-magnet utilization. Using our algorithms retrospectively can help assess prior scheduling decisions to identify potential areas of efficiency improvement as well as identify difficult examination types. Using a year of patient data and several years of MRI utilization data, we construct a simulation model to forecast MRI machine demand under a variety of scenarios. Under our predicted demand model, the throughput calculated by our algorithms acts as an estimate on the MRI time required, and thus, can be used to help predict the impact of different examination trends as well as supporting MRI machine purchase planning.
\end{abstract}

\section{Introduction}

In this paper, we devise models and algorithms to support decision makers at Seattle Children's Hospital $(\mathrm{SCH})$ with the resource allocation and planning problems faced by the Radiology Department concerning MRI examinations. SCH serves pediatric inpatients and outpatients with over 50 specialty clinics. MRI is an imaging modality which uses magnetic fields to help diagnose a wide variety of conditions including tumors, infections, cardiac conditions and musculoskeletal disorders. MRI machines are differentiated by magnet strength, measured in Tesla, and we subsequently refer to MRI machines as magnets. SCH owns one 1.5 Tesla (1.5T) and one 3 Tesla (3T) magnet, which are typical diagnostic strength magnets. Since stronger magnets generate finer detail but also more artifacts, some examination types are best done on the $1.5 \mathrm{~T}$ magnet, others on the $3 \mathrm{~T}$ magnet and still others can be done on either. In addition, each examination type consists of a certain number of mandatory sequences and an additional number of discretionary sequences. Typically, multiple images comprise each sequence. SCH performs MRI examinations on inpatients and outpatients, who might be in an urgent care situation and require an expedited MRI examination.

A critical resource planning question at $\mathrm{SCH}$ has been when to purchase an additional magnet, due to both increasing patient needs and the high expense of the magnets and support staff. In addition, an important goal is to increase magnet utilization during the normal working day (7 AM to $7 \mathrm{PM}$ at $\mathrm{SCH}$ ) while reducing the overtime pay required to meet the patient demand.

Based on interviews with multiple doctors, technologists, and schedulers at SCH we learned the following about the current situation.

\footnotetext{
${ }^{*}$ Science Applications International Corporation adam.capenter@saic.com

$\dagger$ Mathematics Department, The College of William \& Mary, leemis@math.wm.edu.

${ }^{\ddagger}$ Mathematics Department, The College of William \& Mary, papir@math.wm.edu

$\S$ Mathematics Department, The College of William \& Mary, phillips@math.wm.edu. Supported in part by NSF grant DMS-0703532 and a NASA/VSGC New Investigator grant.

『Seattle Chilldren's Hospital, grace.phillips@seattlechildrends.org
} 
- Scheduling MRI patients was already done in an effective, flexible manner that would be difficult to change due to both medical and logistical challenges. In particular, the current process-oriented improvement strategies combined with flexible inpatients allowed sensible rescheduling of patients as needed throughout a given day.

- There was considerable skepticism to the idea that mathematical modeling could make scheduling or purchasing decisions that would be in the interest of both the patients and SCH due to the complexity and uniqueness of individual medical conditions. In addition, there was a reluctance to use reimbursement rates as a metric in determining magnet utilization.

- Although automated data collection of magnet utilization and examination type is already in place, the information was not being used to assess magnet utilization and identify possible areas of scheduling improvement.

Therefore, we provide SCH with a descriptive analysis. In particular, we use optimization models and simulation techniques to identify possible areas of improvement in the current scheduling practices and also predict the impacts of patient examination trends on magnet utilization.

In order to balance both the concerns of the hospital and the patient, we modeled the scheduling problem faced by $\mathrm{SCH}$ as having two competing objectives, magnet overtime utilization versus a fairness metric, which we call fairtime, that measures the magnet time patients receive and is independent of the particular examination type the patient requires. We devise an algorithm to compute tradeoff curves between the two competing objectives. Since there are several other objectives of importance (e.g., revenue), our model and algorithm are meant to be used in conjunction with other information the decision maker possesses.

Our contributions are as follows.

(1) Design a decision support tool to help assess current scheduling practices and identify potential areas of improvement. In particular, our tool is meant to work in conjunction with the process-orientated improvement system in place.

(2) Design a simulation based on current data that, in conjunction with the decision support tool, can be used to perform scenario analysis in possible MRI examination demands. The results of the analysis can then be used to aid in decisions about both personal and magnet planning.

We provide theoretical and practical evidence our algorithm performs well. Our algorithm is provably efficient, but is an approximation to the true optimal solution. We therefore focus on the performance of the objective. Since our problem is computationally difficult to approximate, we derive resource augmentation guarantees in order to measure the theoretical performance of our algorithm. Practical experience on both real and simulated data support that our algorithm finds solutions close to optimality.

We ran the algorithm on a calendar year of patient data from SCH. The data includes demographical information about the patients, examination start times, MRI examination type \& duration, inpatient versus outpatient status and magnet type. ${ }^{1}$ Based on an analysis of the patient data we designed a Monte Carlo simulation to work with the algorithm to predict solutions to the stochastic variant of the scheduling problem. The results of this stochastic optimization problem are then used as predictions for the impact of scenarios, which then led support to purchasing decisions.

We emphasize that our decision support tool is not meant to make scheduling or purchasing decisions. Instead, the tool is designed to provide support for decision makers who wish to identify potential areas of improvement in scheduling or analyze predicted outcomes from possible future trends in MRI examination demand.

The literature on medical appointment scheduling is extensive and we describe only a small sample of work in the area. Further references can be found in the surveys by Cayirli and Veral [3] and Gupta and Denton [9]. Work by Green, Savin, and Wang [8] also analyze the problem of scheduling MRI magnets. They focus on the two related problems of admitting patients into service and outpatient scheduling. They

\footnotetext{
${ }^{1}$ Our data was collected and analyzed with the approval of the SCH Institutional Review Board.
} 
show a particular dynamic policy is optimal under some structural assumptions and provide outpatient scheduling heuristics that perform well in computations. Our work departs from theirs in several respects. They consider profit maximization whereas we seek to simultaneously maximize both regular-hour utilization and a patient fairness metric. They also categorize patients into three categories, outpatients, inpatients, and emergency, whereas we use these categories in addition to the over 100 examination types performed on the MRI magnets. In particular, they assume that patients all require the same magnet time. They also deal with the more difficult problem of determining an optimal online policy whereas we focus on an offline optimal schedule. In part, the latter difference highlights their focus on a prescriptive model versus our focus on a descriptive model.

Also relevant to our problem is the work of Patrick and Puterman, who use scheduling techniques to determine a scheduling policy for outpatient CT-scanner examinations in order to minimize the amount of overtime used [13], which is equivalent to our utilization objective. Our work differs from Patrick and Puterman as they focus solely on developing models and algorithms to improve resource utilization, whereas our models and algorithms help to improve resource planning in addition to resource utilization. In addition, our methods also consider a second objective which measures the time allocated to each patient. Kolisch and Sickinger [11] also consider CT-scanner examination scheduling where they use a Markov Decision Process model to analyze priority policies in order to maximize a revenue-based objective which takes into account the same patient groups as in [8]. The same authors also show a generalization of the classical Bailey-Welch priority rule $[1,17]$ works well when scheduling two CT-scanners (Sickinger and Kolisch [15]). Vermeulen, et al. [16] describe a CT-scanner examination scheduling algorithm that adapts to current and future demands within five patient groups through the use of heuristics and simulation.

We describe a mixed-integer programming formulation and our algorithm in Section 2. We provide an analysis of the patient data in Section ??. In Section 3, we describe our simulation for generating predicted future data. In Section 4, we provide results from our algorithm when used on the data from Section ?? and generated data from Section 3. We conclude with observations on our results in Section 5.

\section{Calculating a fair resource plan}

In this section, we describe an algorithm determines the trade-off between additional magnet hours required and allocating each patient as much time as possible within the schedule assuming the data is deterministic. We then use this algorithm to retrospectively analyze schedules or on a sampling of data generated via simulation. Thus, we assume all the data we are given is deterministic.

Our approach: The optimization problems we encounter are NP-Hard, which indicates that it is unlikely for efficient, exact algorithms to exist (see, e.g., [7]). Moreover, commercial solvers could not solve the mixed-integer programs to a reasonable accuracy even after several hours of computation. To develop effective methods to solve our problems, we consider techniques from approximation algorithms. For $\beta \geq 1$, a $\beta$-approximation algorithm for a minimization problem with optimal objective $v^{*}$ efficiently finds a solution with objective value $\bar{v}$ such that $\bar{v} \leq \beta v^{*}$. However, since the optimization problems we consider can have $v^{*}=0$, finding a $\beta$-approximation algorithm is as difficult as finding an exact algorithm. We address this differently by employing resource augmentation techniques [10]. For $\gamma \geq 1$, we define a $\gamma$-speed scheduling algorithm to be one which finds a solution with objective $\bar{v} \leq v^{*}$ assuming all process times were scaled by $\gamma$, e.g., if $p_{i j}$ represent the processing time required by a job $j$ on machine $i$, then a $\gamma$-speed algorithm sets $p_{i j} \leftarrow \frac{p_{i j}}{\gamma}$, i.e., all machines were "sped up" by a factor of $\gamma$. Such an approach is natural in our situation because we are interested in minimizing the amount of extra magnet time required. Note that 1-approximation algorithms find the exact optimal solution, as do 1-speed algorithms.

Mathematical formulation: We now describe a mathematical programming formulation of our problem. We make the following assumptions. 
1. The schedulers at SCH currently allocate examinations into 45-minute time slots. However, the technologists use such schedules as guidelines and make decisions throughout the day to accommodate patients' actual varying examination times. Therefore, the schedule used at SCH is actually flexible and the optimization tool only schedules to the day, rather than in 45-minute time slots. For scheduled patients, we allow the optimizer to reschedule the appointment to any day within two days of their simulated scheduled appointment. We assume unscheduled patients must be seen on the day they arrive into the system.

2. We assume that the magnet that the patient is scheduled on is fixed since such a decision also involves diagnostic, as well as logistic, determinations.

3. For a given MRI examination on a given magnet, there are also a number of discretionary sequences that can be performed in addition to those required by the examination type. Therefore, we assume that each exam has a magnet-specific minimum amount of operation time required and a magnet-specific maximum amount of operation time that could be used.

Minimizing magnet operation-time involves determining the operation-time allocated for a specific exam conducted on a specific magnet. If all SCH wished to do was maximize the throughput of examinations, the scheduling algorithm should allocate every patient the minimum magnet time required. However, such an allocation ignores the diagnostic value of the discretionary sequences, and so we also create a second objective in order to maximize the minimum normalized time each exam-magnet combination receives. Enumerate all the examinations that must be run. For a given examination $j$, let $\ell_{j}$ and $u_{j}$ denote the minimum and maximum of magnet time required, respectively, and $p_{j}$ be the decision variable indicating the amount of magnet time allocated. Thus, $\ell_{j} \leq p_{j} \leq u_{j}$. We define

$$
\varphi_{j}=\frac{p_{j}-\ell_{j}}{u_{j}-\ell_{j}}
$$

as the fairtime of examination $j$ and define fairtime as the minimum fairtime over all examinations, i.e., $\varphi=\min _{j} \varphi_{j}$

Below is an exact formulation to schedule examinations on one magnet while simultaneously minimizing the maximum extra magnet time required over all days and maximizing the fairtime.

$$
\begin{array}{lll}
\min / \max & \{F, \varphi\} & \\
\text { subject to } & \sum_{t} x_{j t}=1 & \forall j \\
& \sum_{j} p_{j} x_{j t} \leq b+F_{t}, & \forall t \\
& F_{t} \leq F, & \forall t \\
& \ell_{j} \leq p_{j} \leq u_{j} & \forall j \\
& \varphi_{j}=\frac{1}{u_{j}-\ell_{j}}\left(p_{j}-\ell_{j}\right) & \forall j \\
& \varphi_{j} \geq \varphi & \forall j \\
& x_{j t}=0 & t \notin\left\{r_{j}, \ldots, d_{j}\right\} \\
& F, F_{t}, p_{j} \geq 0, x_{j t} \in\{0,1\}, & \forall j, t,
\end{array}
$$

where the problem parameters are

$r_{j}, d_{j}, \quad$ the earliest and latest day examination $j$ can be conducted,

$b$, the minutes each magnet is available for each day,

and the decision variables are

$p_{j}, \quad$ the minutes allocated to examination $j$.

$x_{j t}$, indicates that examination $j$ is on day $t$.

$F_{t}, \quad$ the extra minutes allocated to the magnet on day $t$.

$F, \quad$ the maximum amount of minutes allocated over all days.

Constraints (a) ensure that each examination is conducted. Constraints (b) ensure that the magnet is allocated enough minutes on a given day $t$ to conduct all examinations. Constraints (c) ensure that the 
maximum $F_{t}$ is being minimized. Constraints (d) enforce the upper and lower bound on the examination time allocated to examination $j$. Constraints (e) calculate the fairtime for examination $j$. Constraints (f) ensure the minimum fairtime is allocated and constraints $(\mathrm{g})$ ensure that examinations are only scheduled on feasible days. Note that (2) is a quadratic mixed-integer bicriteria program and is generally difficult to solve as modelled. However, the following proposition simplifies the problem. In particular, the following proposition shows that if an optimal solution exists for (2), then an optimal solution exists where $\bar{\varphi}_{j}$ are all the same.

Proposition 1. Suppose $\left(x_{j t}, p_{j}, \varphi_{j}, \varphi, F_{t}, F\right)$ is a feasible solution vector to (2). The solution, $\left(x_{j t}, \bar{p}_{j}, \bar{\varphi}_{j}, \varphi, F_{t}, F\right)$ is also feasible where

$$
\bar{p}_{j}=\ell_{j}+\varphi\left(u_{j}-\ell_{j}\right), \forall j, \quad \bar{\varphi}_{j}=\varphi, \forall j .
$$

In particular, the two solutions have the same objective.

Proof. Constraints (a), (c), (d), (e) and(f) are all satisfied by definition. To see that constraint (b) is satisfied, note that $\bar{\varphi}_{j}=\varphi \leq \varphi_{j}$ for all $j$, which implies $\bar{p}_{j} \leq p_{j}$. Since $x_{j t} \geq 0$,

$$
\sum_{j} \bar{p}_{j} x_{j t} \leq \sum_{j} p_{j} x_{j t} \leq b_{t}+F_{t}, \forall t
$$

Thus, all constraints are satisfied.

So, to make the problem linear, we can instead consider the following two families of (linear) integer programs (IP).

$$
\begin{array}{llll}
G(\varphi)=\min & F & & \\
\text { subject to } & \sum_{t} x_{j t}=1 & \left(a_{\varphi}\right) \\
& \sum_{j} p_{j t} x_{j t} \leq b+F_{t}, & \forall t & \left(b_{\varphi}\right) \\
& F_{t} \leq F, & \forall t & \\
& x_{j t} \in\{0,1\} & \forall j, t,
\end{array}
$$

where for all $j$ and $t$,

$$
p_{j t}= \begin{cases}\ell_{j}+\varphi\left(u_{j}-\ell_{j}\right), & t \in\left\{r_{j}, \ldots, d_{j}\right\}, \\ M, & \text { otherwise. }\end{cases}
$$

Solving (3) is NP-Hard, but we can relax the integrality constraints $x_{j t} \in\{0,1\}$ to $0 \leq x_{j t} \geq 1$ to create a solvable model. We attempted to solve (3) using CPLEX 11 on two dual-core Opteron $2.6 \mathrm{Ghz}$ processors with 8 GB of memory but did not close the integrality gap to within $10 \%$ even after 24 hours of computation. Once the LP relaxation of $G(\varphi)$ is solved we can then solve the LP relaxation of the following IP.

$$
\begin{array}{llll}
H(\varphi)=\min & \sum_{j, t} t x_{j t} & & \\
\text { subject to } & \sum_{t}^{t} x_{j t}=1 & \forall j & \left(a_{\varphi}\right) \\
& \sum_{j} p_{j t} x_{j t} \leq b+G(\varphi), & \forall t & \left(b_{\varphi}\right) \\
& x_{j t} \in\{0,1\} & \forall j, t . &
\end{array}
$$

We denote $G^{L P}(\varphi)$ and $H^{L P}(\varphi)$ as the LP relaxations of $G(\varphi)$ and $H(\varphi)$ respectively. Note that $G^{L P}(\varphi) \leq$ $G(\varphi)$ and $H^{L P}(\varphi) \leq H(\varphi)$. Also, all optimal solutions to $G(\varphi)$ are also feasible to $H(\varphi)$ and all optimal solutions to $G^{L P}(\varphi)$ are feasible to $H^{L P}(\varphi)$. Also, in $H^{L P}$, the objective is equivalent to minimizing the average flowtime for all examination, i.e., the average number of days from the release day to the day when the examination is completed. For a given $\varphi$, holding all $p_{j t}$ to the "minimum" $\varphi$ for permissible days is without loss due to Proposition 1. Note that solving for $G(\varphi)$ is the computational bottleneck of the FindTradeofF algorithm (see Algorithm 1. By considering each day as "machine," (3) is actually 


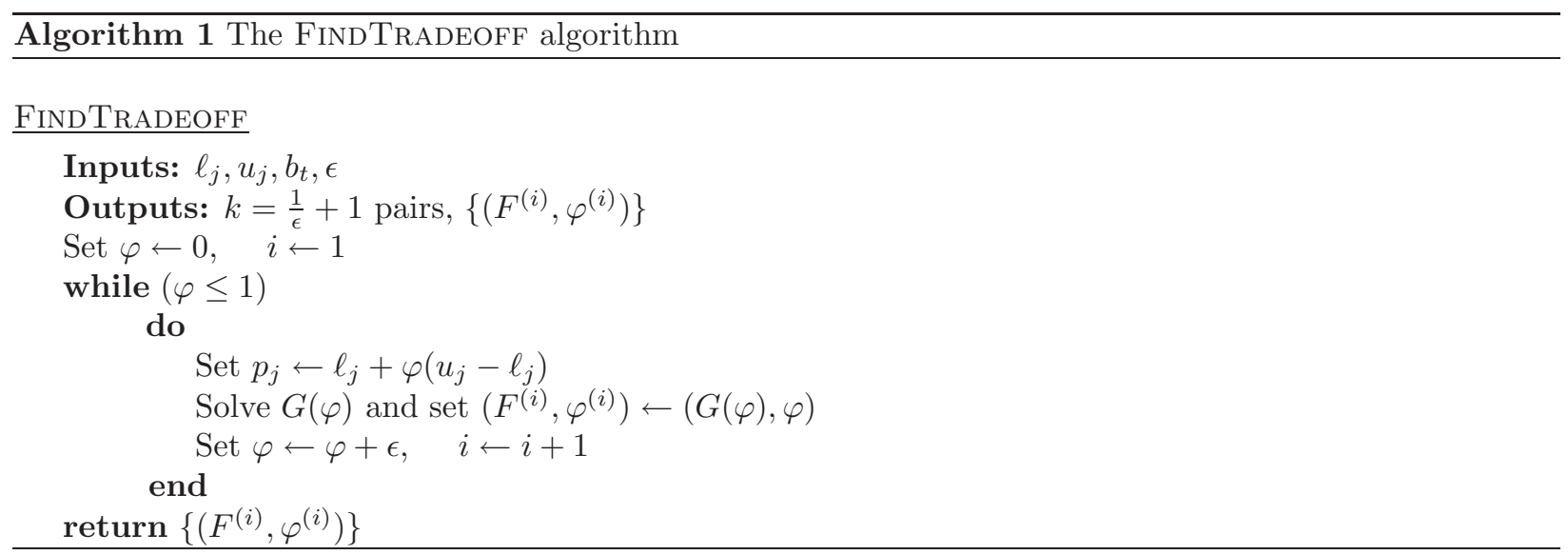

a problem of minimizing the maximum tardiness where all jobs have an identical due date of $b$ and the machines are unrelated. However, such a scheduling problem is at least as hard as finding the minimum makespan $^{2}$ on identical parallel machines, which is NP-Hard [6]. Such scheduling problems are also known to be difficult for integer programming software to solve. Moreover, for our problem, if $b$ is set to the optimal makespan i.e., the minimum $\max _{t} \sum_{j} p_{j} x_{j t}$ possible for any schedule, then the optimum maximum tardiness is zero. Thus, no $\beta$-approximation algorithm exists if $P \neq N P$ since any $\beta$-approximate solution is identical to the true optimum. Thus, we focus on finding a $\gamma$-speed scheduling algorithm. We use an algorithm of Shmoys and Tardos [14] that converts solutions of the LP relaxation of $G(\varphi)$ into integral solutions by solving a minimum cost matching problem on an associated bipartite network. The key theorem regarding the objective guarantee is as follows.

Theorem 2 (Shmoys and Tardos [14]). A solution, $\mathbf{x}$, to $H^{L P}(\varphi)$ can be converted into a (integral) solution, $\overline{\mathbf{x}}$, to $H(\varphi)$ with $\sum_{j t} t \bar{x}_{j t}=H^{L P}(\varphi)$ and constraint $\left(b_{\varphi}\right)$ of (4) satisfied so that

$$
\sum_{j} \bar{x}_{j t} \leq b+G^{L P}(\varphi)+p_{\max }
$$

where $p_{\max }=\max _{j, t}\left\{p_{j t}: t \in\left\{r_{j}, \ldots, d_{j}\right\}\right\}$.

As a corollary, we can state the following.

Corollary 1. There is an algorithm that can find a (integral) solution $\overline{\mathbf{x}}$ to $G(\varphi)$ with $\sum_{j, t} t \bar{x}_{j t} \leq H^{L P}(\varphi)$ and objective $\bar{F}$ so that $\bar{F} \leq G(\varphi)+p_{\max }$ where $p_{\max }=\max _{j, t}\left\{p_{j t}: t \in\left\{r_{j}, \ldots, d_{j}\right\}\right\}$. Moreover, such $a$ solution can be found in time proportionate to solving the $G^{L P}$ and $H^{L P}$.

Proof. Suppose that $\mathbf{x}$ is a solution to $G^{L P}(\varphi)$. Then $\mathbf{x}$ is feasible to $H^{L P}$ and we can find the optimal solution, $\mathbf{x}^{*}$, to $H^{L P}$. We can then find an integral solution $\overline{\mathbf{x}}$ to $H(\varphi)$ by Theorem 2 with

$$
\sum_{j} \bar{x}_{j t} \leq b+G^{L P}(\varphi)+p_{\max }
$$

Clearly, $(\overline{\mathbf{x}}, \bar{F})$ is feasible to $G(\varphi)$ with $\bar{F} \leq G^{L P}(\varphi)+p_{\max }$.

With respect to our problem, the maximum examination length is known to be no more than some small fraction of the daily magnetic availability. We denote this fraction by $\alpha$. Then we can state the following theorem as a consequence of Theorem 2 .

\footnotetext{
${ }^{2}$ The makespan is the maximum completion time for any job.
} 
Theorem 3. Suppose there is some $\alpha \in(0,1)$ so that $p_{\max } \leq \alpha b$. There is a $(\alpha+1)$-speed algorithm to find integral solutions to $G(\varphi)$.

Proof. Let $\varphi$ be given. Using Corollary 1, solving $G^{L P}(\varphi), H^{L P}(\varphi)$ and applying Shmoys-Tardos will generate a solution $\left(\overline{\mathbf{x}}, \bar{F}, \bar{F}_{t}\right)$ to $G(\varphi)$. Let $\bar{C}$ denote the makespan of the schedule generated by the ShmoysTardos algorithm and $C^{*}$ denote the optimal makespan, i.e., $\bar{C}=\max _{t}\left\{b+\bar{F}_{t}\right\}=b+\bar{F}$ and $C^{*}=b+F^{*}$. Then, by Corollary 1, we have that

$$
\bar{C}=b+\bar{F} \leq b+F^{*}+\alpha b=C^{*}+\alpha b .
$$

Now consider the same solution if each machine's "speed" was increased by a factor of $(\alpha+1)$, which is equivalent to setting all process times to $\frac{1}{\alpha+1} p_{j t}$. Thus, at this speed, the approximate solution has completion time

$$
\bar{C}^{S}=\frac{1}{1+\alpha} \bar{C} \leq \frac{1}{1+\alpha}\left(C^{*}+\alpha b\right) .
$$

Then the objective on the faster machines is

$$
\bar{F}^{S}=\left(\frac{1}{1+\alpha} \bar{C}-b\right)^{+} \leq\left(\frac{1}{1+\alpha}\left(C^{*}+\alpha b\right)-b\right)^{+}=\left(\frac{1}{1+\alpha}\left(C^{*}-b\right)\right)^{+} \leq\left(C^{*}-b\right)^{+}=F^{*} .
$$

So, in our implementation of FindTradeoff, we solve $G(\varphi)$ approximately. An interesting feature of our method is that we also return a solution with optimal average flowtime. In particular, we have the following corollary.

Corollary 2. The algorithm FINDTRADEOFF, when implemented via Theorem 3, is a $(\alpha+1)$-speed algorithm to the bicriteria problem of $G(\varphi)$ where both $F$ and average flowtime are minimized.

We note that we could use Theorem 2 on the first LP solution. In Section 4 we examine the benefit of using the second over the first.

\section{Simulating schedules}

In this section, we describe our simulation model used to generate inputs for FINDTRADEOFF. Since we seek to simulate the MRI examination demand at $\mathrm{SCH}$, we first conduct a analysis of our data sets. In this study, we use both MRI patient data (the patient dataset) and weekday examination counts (the trend dataset) from SCH radiology department. We describe and analyze the patient dataset in Sections 3.1 and 3.2 and the trend dataset in Section 3.3 below. We describe our simulation model in Section 3.4.

Our data analysis and simulation model was written in S-Plus and performed on two dual-core Operon $2.6 \mathrm{GHz}$ processors with $8 \mathrm{~GB}$ of RAM.

\subsection{Collecting the patient dataset}

The patient dataset is composed of 5,917 MRI examinations conducted from July 1, 2007 to June 30, 2008 at $\mathrm{SCH}$ and consists of two basic types of examinations. Not included in the dataset were 17 records for reasons described below. Patients who made examination appointment times through the schedulers at SCH are referred to as scheduled patients. Scheduled patients' appointment times were recorded in the schedulers' computer system. Patients who did not schedule examination appointment times and were added on an ad hoc basis to the preexisting schedule by the technologists are referred to as unscheduled patients.

Depending on their condition, previous examination history, and diagnosis, patients received varying amounts of pre-examination preparation and were examined on either the $1.5 \mathrm{~T}$ or $3 \mathrm{~T}$ magnets. Some exams require that a patient receive intravenous contrast and/or anesthesia in order to receive a satisfying 
examination. In addition, preparation time for both the patient and magnet was necessary for the magnet used. The amount of patient and magnet preparation time required prior to an exam was not recorded, although the need for intravenous contrast and anesthesia was.

A magnet is considered to be in operation from the time the first image is acquired (magnet on-time) until the time the last image is acquired (magnet off-time). Both magnet on-time and off-time were automatically recorded by the computers used to operate the magnets and magnet operation-time was calculated as the interval between magnet off-time and magnet on-time. During the examination, the technologist recorded additional information including the exact exam type performed. After the completion of an exam, a radiologist interpreted the results and dictated a report. A radiologist and an undergraduate assistant combined these separate computer records and encrypted any personal patient information as per IRB specifications. The patient data was imported into S-PLUS, a statistical computer package. All data fields are described in Appendix A.1.

Throughout the course of the year-long period, the Radiology Department performed 5,934 examinations, of which 17 were omitted and 5,917 included in our study. The 17 patient records omitted, lacked crucial data fields (nine records), appeared to have incorrect data fields (six records), or corresponded to patients too old to be considered representative of the pediatric population (two records). A complete list of the 17 omitted records with justifications can be found in Appendix A.2.

\subsection{Data analysis of the patient dataset}

The initial analysis of the patient dataset yields several trends in patient arrivals and magnet operation-times.

1. There is often a strong preference to perform certain examination types on a specific magnet type.

2. Magnet operation-time distributions differ depending on examination type, magnet type, and scheduled status.

3. The volume of unscheduled patients examined varies throughout the day, exhibiting similar behavior, but at different magnitudes, for each weekday and magnet type.

SCH Radiology Department performed 112 different types of MRI examinations during the study period. The proportion of male to female patients was roughly equal in the 5,917 examination records (51.7\% male). Unscheduled patients accounted for a significant fraction of the examinations performed (19.7\%). The 1.5T magnet was utilized approximately 50 percent more than the $3 \mathrm{~T}$ magnet with respect to both the number of examinations $(59.0 \%$ on the $1.5 \mathrm{~T})$ and the amount of magnet operation-time $(60.8 \%$ on the $1.5 \mathrm{~T})$. The vast majority of MRI examinations conducted were on children (99.9\% under age 18). The ages of patients in the data ranged from one day to 25 years, with a median age of 9.58 years.

For most examination types, the data indicates a strong preference for a particular magnet. However a magnet preference that is apparent for scheduled examinations was not apparent among unscheduled examinations. ${ }^{3}$

The amount of magnet operation-time required for an examination is dependent upon the type of examination, the magnet on which the examination is performed, and the scheduling status of the examination. This implies that the median and variance of magnet operation-time differs not only between examination types, but also between magnet type and scheduled status. These trends are graphically represented in Figure 1. Thus, the distribution of magnet operation-times for a single examination type may exhibit different behavior depending on scheduled status and magnet type.

To further analyze the magnet operation-time, we performed a statistical analysis using a Cox Proportional Hazards (CPH) model [4]. We examined a large subset of the patient dataset (3901 records) corresponding to the five most common examination types, so that the effects of examination type would be accounted for with a sufficient number of observations. Table 1 gives the results of our CPH analysis. In a $\mathrm{CPH}$ model, a log-linear function is used to estimate the covariates effect on the examination time is. A

\footnotetext{
${ }^{3}$ We suspect magnet availability is a mitigating factor.
} 
positive coefficient implies that as a single covariate changes (and all others are held constant) from a lower to a higher level, the magnet operation-time decreases on average. Similarly, a negative coefficient implies that examinations should take longer on average moving from a lower to a higher level for an individual covariate. The results in Figure 1 are listed in decreasing order of significance with covariates satisfying a $p<.05$ level of significance. The results of the $\mathrm{CPH}$ indicate that examination type can play a significant role in determining magnet operation-time, as can contrast, inpatient status, magnet type and scheduled status.
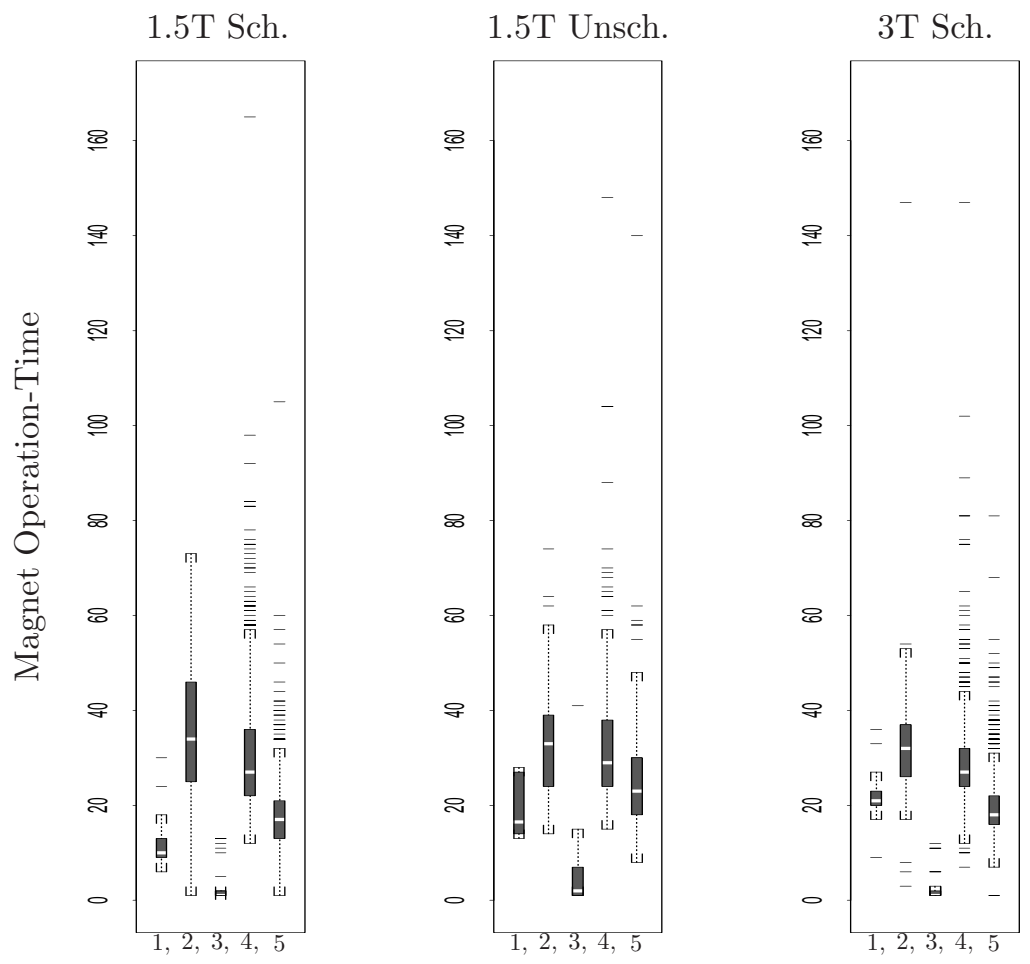

3T Unsch.

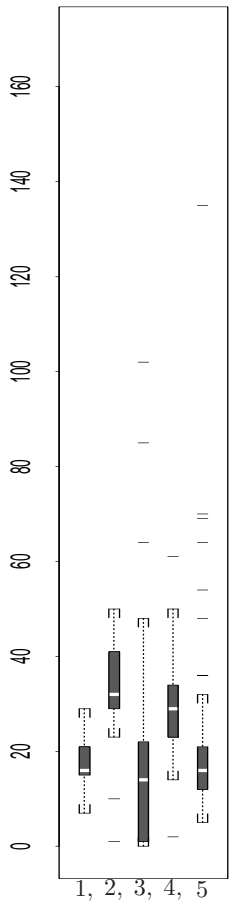

Figure 1: Magnet Operation-Times by Magnet and Examination Type. Shaded boxes represent the middle $50 \%$ of magnet operation-times, with the white line demarcating the median. The whiskers extend out from the 25 th and 75 th percentiles to the most extreme value still within 1.5 times the range of the middle $50 \%$ of the data. The examination types plotted above represent all types with at least 50 examinations in an individual category. The single zero magnet operation-time present is due to rounding. The examination types are listed below.

\begin{tabular}{clcl}
\hline Exam & Examination Type & Exam & Examination Type \\
\hline 1 & Abdomen without contrast & 2 & Angio head without contrast \\
3 & Brain limited & 4 & Brain with and without contrast \\
5 & Brain without contrast & & \\
\hline
\end{tabular}

Using the magnet on-times as estimates for daily arrival rates, we found that the average arrival rates of unscheduled patients vary depending on the day of the week, with Mondays and Fridays having the most unscheduled arrivals to both magnets. Also, as shown in Figure 2, the average arrival rates for the $1.5 \mathrm{~T}$ magnet were consistently higher than for the $3 \mathrm{~T}$ magnet, both across individual weekdays and in the aggregate. Despite having the most unscheduled cases on Mondays and Fridays, the Radiology Department performed the highest number of examinations on Wednesdays (see Figure 3). This implies that Wednesdays also have the most scheduled examinations on average. The data also suggests a increasing trend across the 


\begin{tabular}{lrrrrr}
\hline Covariate (low value, high value) & coef & Exp(coef) & SE(coef) & z-score & p-value \\
\hline Brain without contrast & -0.57106 & 0.565 & 0.0361 & -15.827 & $0.0 \mathrm{e}+00$ \\
Spine total without contrast & -0.39959 & 0.671 & 0.0436 & -9.174 & $0.0 \mathrm{e}+00$ \\
Brain with and without contrast & -0.45485 & 0.635 & 0.1071 & -4.247 & $2.2 \mathrm{e}-05$ \\
Contrast dye status (used, not) & 0.37663 & 1.457 & 0.1050 & 3.589 & $3.3 \mathrm{e}-04$ \\
Outpatient status (out, in) & -0.08983 & 0.914 & 0.0293 & -3.063 & $2.2 \mathrm{e}-03$ \\
Magnet type (1.5T, 3T) & 0.04757 & 1.049 & 0.0182 & 2.618 & $8.8 \mathrm{e}-03$ \\
Spine total with and without contrast & -0.26772 & 0.765 & 0.1111 & -2.409 & $1.6 \mathrm{e}-02$ \\
Scheduled status (scheduled, unscheduled) & -0.06495 & 0.937 & 0.0285 & -2.280 & $2.3 \mathrm{e}-02$ \\
\hline Repeat examination status (repeat, new) & -0.02975 & 0.971 & 0.0175 & -1.699 & $8.9 \mathrm{e}-02$ \\
Priority status (priority, not) & -0.04411 & 0.957 & 0.0375 & -1.175 & $2.4 \mathrm{e}-01$ \\
Gender (male,female) & -0.01368 & 0.986 & 0.0162 & -0.842 & $4.0 \mathrm{e}-01$ \\
Anesthesia status (used, not used) & -0.01978 & 0.980 & 0.0244 & -0.811 & $4.2 \mathrm{e}-01$ \\
Age (years) & 0.00046 & 1.000 & 0.0040 & 0.114 & $9.1 \mathrm{e}-01$ \\
\hline
\end{tabular}

Table 1: The results of the Cox Proportional Hazards model analysis of examination lengths on a large subsection of data (3901 examinations). For each binary, non-numerical covariate, we list our designations of the 'high' and 'low' settings. The entries are sorted in decreasing level of significance. The excluded examination was MR Angio Head without Contrast

year in the average number of examinations performed daily. We note that the trend is supported by regression analyses on the trend dataset, as described below in Section 3.3 (see Figure 4).

\subsection{The trend dataset analysis}

The trend dataset consisted of the total number of examinations per weekday dating from January 1, 2003 to January 31, 2009. As of January 1, 2003, SCH operated a single 1.5T magnet. On October 14, 2006 a $3 \mathrm{~T}$ magnet was installed and the $1.5 \mathrm{~T}$ magnet was replaced with a newer $1.5 \mathrm{~T}$ magnet. We removed 71 weekdays from our data set on which the MRI magnets were not fully operational, such as holidays, staff training days, or days on which maintenance or installation occurred. Appendix A.2 contains a detailed description of the days we removed.

In order to determine the trend in MRI examinations, we regressed the number of daily MRI examinations on the weekdays elapsed in the trend dataset. Since the number and type of available magnets changed, we performed two separate linear regressions. One regression was performed on the weekdays from January 1, 2003 to October 14, 2006, and the other was performed on weekdays from October 14, 2006 until January 31,2009 . The slope found on the first regression was $0.0048(p<0.001)$ and on the second regression was $0.0114(p<0.001)$. The variance in the MRI examinations explained by the weekdays elapsed was similar for both regressions ( $r^{2}=0.1565$ for the first, $r^{2}=0.1738$ for the second), although the overall variance was high.

\subsection{The simulation model}

In order to simulate the number of patients requiring MRI examinations, we consider the arrival process of unscheduled patients and the distribution of scheduled patients. We assume that the unscheduled and scheduled patients are distributed independently and model each population separately. Scheduled patients call the scheduler's office to obtain an appointment for their MRI examination. The scheduler then allocates a time in the schedule that was mutually acceptable to the hospital and the patient. Appointments such as these may be scheduled months in advance. Examinations performed on unscheduled patients, in contrast, are much harder to plan for. Unscheduled patients may arrive to the Radiology Department from other 


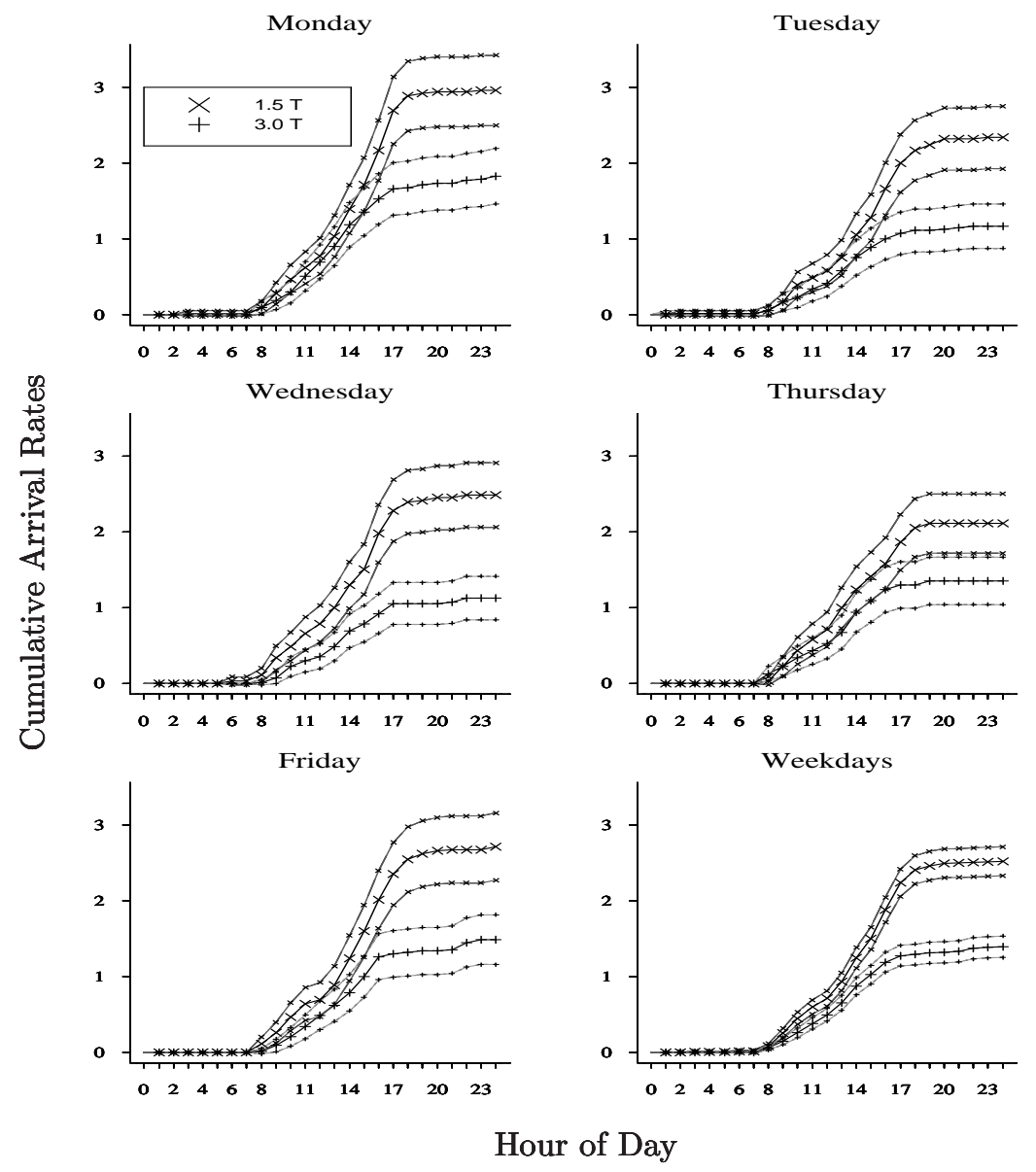

Figure 2: Magnet specific cumulative arrival rates. The cumulative arrival rates to each magnet, by day, with linear interpolation, with associated 95\% confidence intervals. The vertical axes represent the number of patients arriving on average while the horizontal axes represent the time of day, from midnight to midnight. The larger marks represent the average arrival rates while the smaller marks represent the bounds of the $95 \%$ confidence intervals.

departments within SCH or from outside clinics or hospitals with little to no warning, and may require immediate attention. Therefore, we also model the priority status for patients which indicates how soon the patient must undergo an MRI examination.

Modeling the unscheduled patient arrival process relied heavily on the use of magnet on-times to approximate arrival times. As shown in Figure 3, unscheduled patients do not arrive at a constant rate. Arrivals are infrequent during the night and early morning hours, increase dramatically during the afternoon, and subsequently decrease to nighttime levels. Arrival rates also differ by day of the week and magnet type (Figures 2 and 3). Since SCH only records magnet on-time, and no more than one examination can be performed at a time on a given magnet, it is nearly impossible to determine the frequency with which groups of two or more simultaneous patients arrive from our data. Anecdotal evidence suggests that group arrivals are infrequent, and so we assume that patients arrive singly. Given this information, we model the arrival of unscheduled patients as a non-homogeneous Poisson process. A Poisson process assumes that the chance of an arrival in a given time interval only depends on the average arrival rate and length of the time interval. A non-homogeneous Poisson process has time-dependent arrival rates. Average arrival rates were calculated 
Unscheduled, 1.5T Arrivals
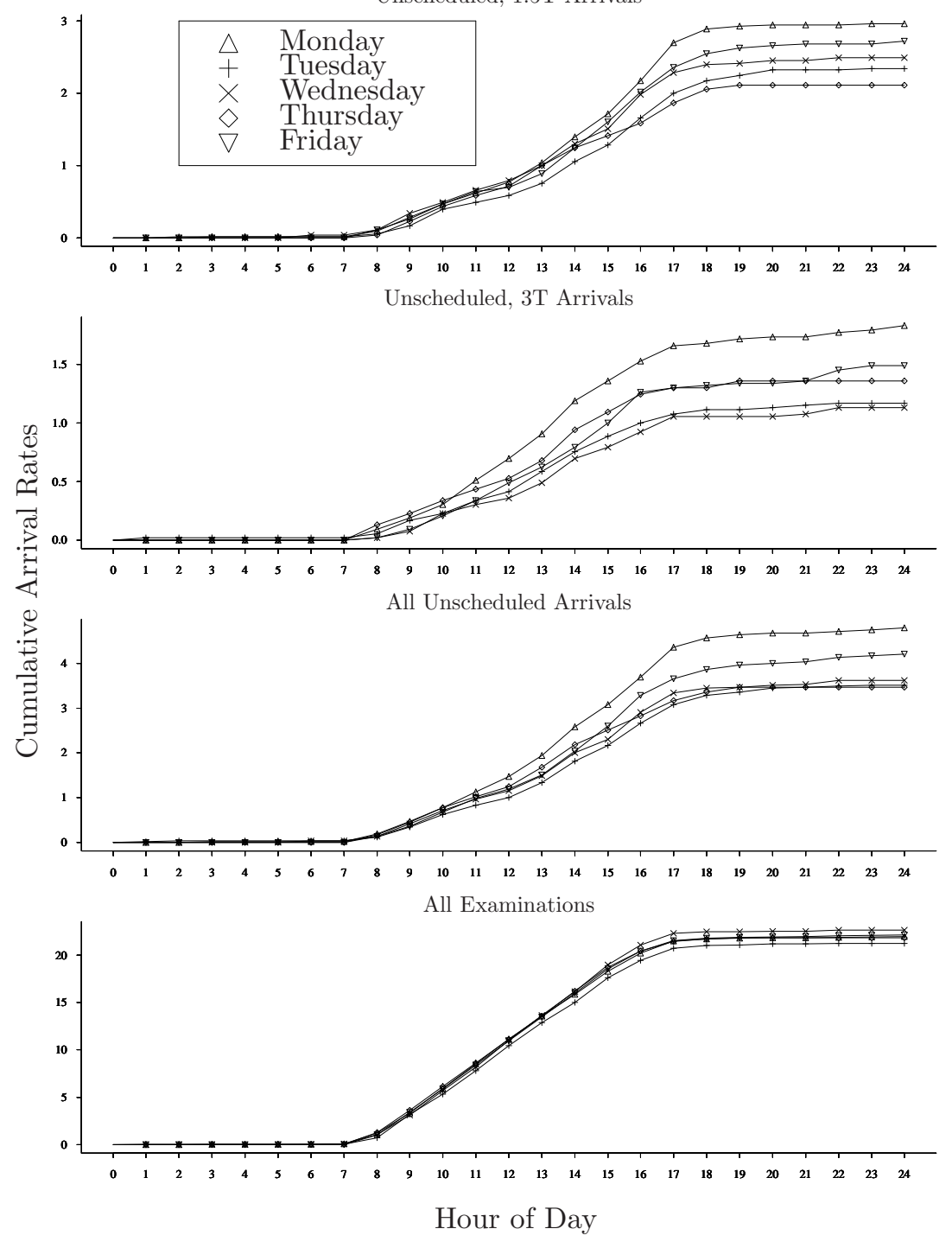

Figure 3: The cumulative arrival rates by day. The first two plots show the cumulative arrival rates of unscheduled examinations to the $1.5 \mathrm{~T}$ magnet and the $3 \mathrm{~T}$ magnet respectively. The third plot shows the cumulative arrival rates of unscheduled examinations to both magnets, and the fourth plot displays how the average total number of examinations increases over the course of a day.

for each hour of each weekday. For more information about non-homogeneous Poisson processes, including applications, see [12].

Once the arrival time of an unscheduled patient had been generated, we independently and randomly sample examinations from the data recorded from July 1, 2007 to June 30, 2008, using the examination type, priority status, contrast and anesthesia requirements and magnet operation time of an examination record as the simulated patients needs. This method is a simple way to incorporate the underlying correlations of exam traits to be represented in our simulated data. By doing so, we are assuming that the examination type required by unscheduled arrivals are independent of time of day and day of week. Such an assumption was reasonable in light of the small proportion of unscheduled arrivals. 

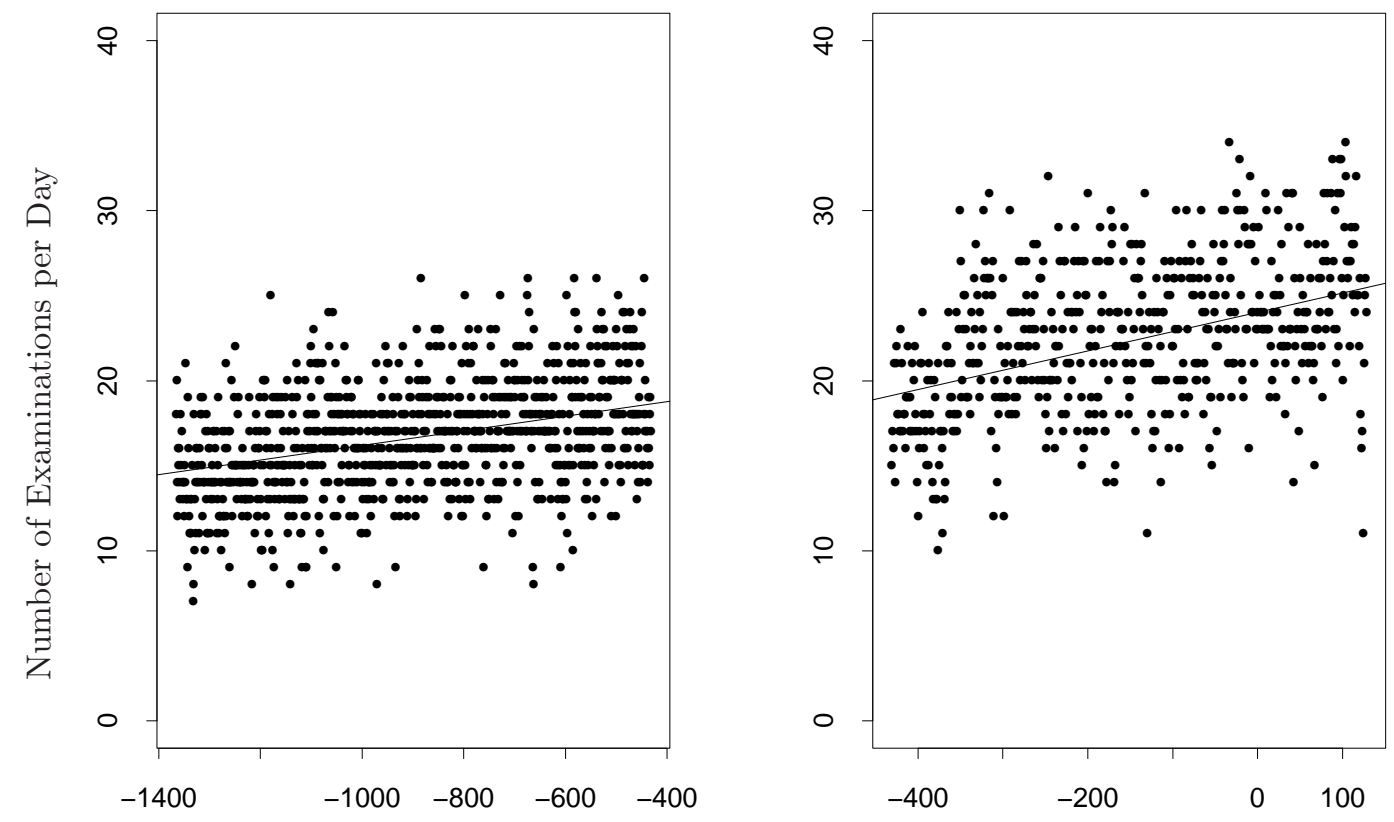

Days after June 30, 2008

Figure 4: The single magnet data and regression line (left) and the two-magnet data and regression line (right). The horizontal axis measures the days in our trend dataset with day 0 representing June 30, 2008, the last day of the patient dataset. The single magnet plot ends the day of the installation of the 3T magnet, and the two-magnet plot begins the day after the installation.

We took a different approach in modeling the distribution of patients requiring scheduled examinations. We do not have information regarding when patients first contacted the scheduling office. Instead, we use the magnet on-times and examination dates of scheduled examinations in order to model the distribution of MRI examinations amongst the scheduled patients. We generate the examination information for a day by randomly selecting a weekday from the patient data set, using the scheduled examinations from that day as the examinations requiring scheduling. We also use the actual magnet operation-time as simulated operation time in order to account for codependencies in magnet operation-time and the examination type.

Since the simulation is used to model future patient load of the MRI magnets, we use the slope of the second regression as the trend in increased MRI examinations. Since schedulers can, ostensibly, control the number of scheduled MRI examinations, we assume that the increase in examinations was all in unscheduled patients. ${ }^{4}$ Thus, each day of the simulation, the arrival rate of unscheduled patients was increased by the slope of the second regression (i.e., 0.0114). As the linear regression applies a reasonable fit, we assume that the average arrival rate over the patient data set time period is equivalent to the arrival rate at the middle of that time period (i.e., December 30, 2007). Thus, we assume that the daily arrival rates at the beginning of the study are 1.482 larger than the average arrival rates over the patient data set time period. The simulation begins the day after the end of our study period, July 1, 2008, and can generate examination

\footnotetext{
${ }^{4}$ We note that the increase could also be due to other factors such as improved efficiency in conducting examinations or an increased proportion of shorter MRI examinations required.
} 
Tradeoff CURVes Between $\varphi$ AND OVERTIME
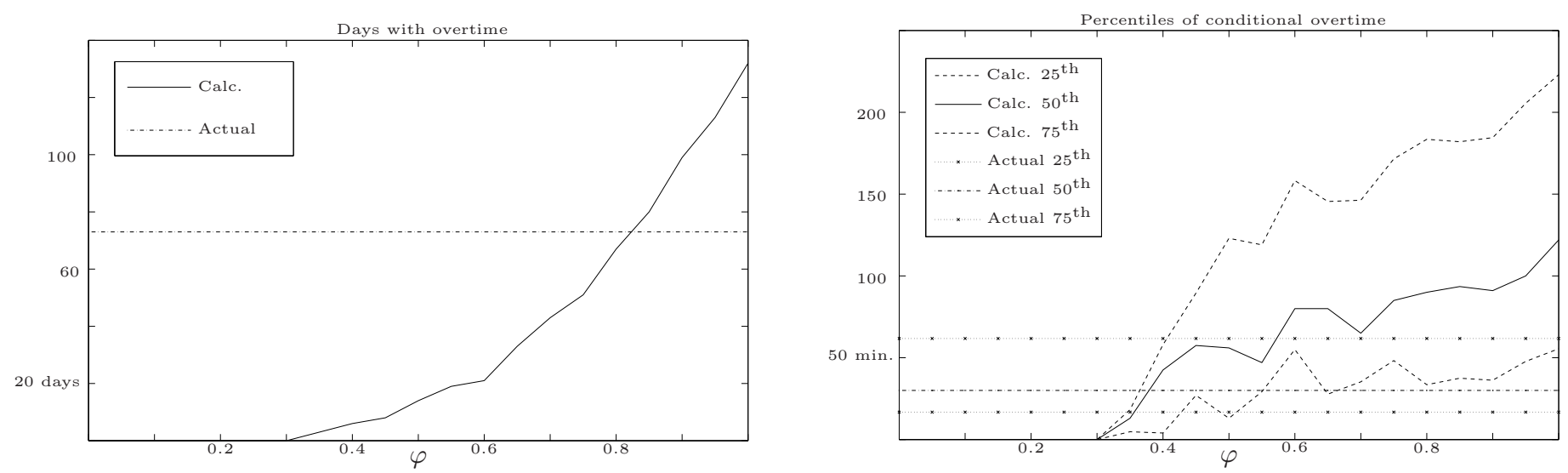

Figure 5: These results were generated when FindTRADEOFF was run on the real data for the 1.5T magnet. The graph on the left displays the tradeoff between the percentage of allocated examination time $(\varphi)$ and days with overtime. The graph on the right displays the tradeoff between percentage of allocated examination time and the quartiles of the conditional overtime. The horizontal lines are the quartiles from the actual overtime that occurred.

data over an arbitrarily long time period.

\section{Results}

In addition to aiding in planning for the next magnet purchase, our tool can be used to help identify potential improvements in scheduling and/or resource allocation. In Section 4.1, we discuss how the latter can be done by running the scheduling algorithm on the real data and making a comparison to the actual schedule. In Section 4.2, we describe how the algorithm and simulation models can be used to help forecast and plan for a new magnet purchase. Since the utilization is higher on the 1.5T, we present results only for this magnet. Our methods can be used identically on the $3 \mathrm{~T}$ magnet.

For our aggregate measures, we focused on yearly magnet overtime minutes. We define magnet overtime as the amount of magnet time that occurred outside normal operating hours, which were from 7 AM to 7 PM. When measuring the magnet overtime minutes for a year, applying a statistic (e.g., average, median, etc.) without removing the days where no magnet overtime occurred artificially skews the statistic based on the incidence of magnet overtime. For example, suppose 2009 had two days with magnet overtime of 50 minutes in each day, and in 2010 there were 20 days with magnet overtime of 50 minutes in each day. The ordinary average overtime in 2009 (assuming 250 workdays) is 0.2 minutes and 4 minutes in 2010. However, this masks the actual change: the conditional average magnet overtime in both years was 50 minutes whereas the incidence sharply rose from 2 days out of 250 to 20 days out of 250 . Therefore, we examined the incidence and the severity separately. Our chief metrics were to examine the incidence of days with magnet overtime and the conditional magnet overtime minutes, i.e., the average magnet overtime minutes over days when an overtime event occurred.

FindTRADEOFF predicts lower bounds on the amount of overtime minutes for the following reasons.

- FindTradeOfF works with magnet ontimes only - thus, there are no setup times.

- FindTradeoff is allowed to schedule all patients, scheduled or unscheduled, within two days of their originally simulated examination date. 
DAILY COMPARISON BETWEEN OVERTIME MINUTES FROM THE ALGORITHM VS. THE ACTUAL SCHEDULE
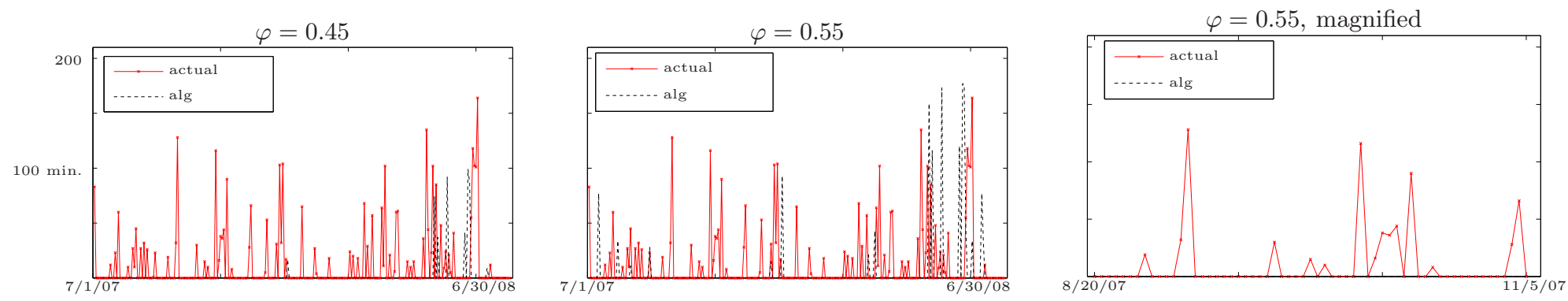

Figure 6: The first two graphs compare the schedule from FindTRADEOFF to actual schedule on the 1.5T magnet when varying $\varphi$ from 0.45 to 0.55 , which increases the total minutes scheduled from 118,294 to 131,592. The original schedule contained 94,465 minutes of magnet ontime. The comparison between the algorithmically generated schedule and the actual schedule can be used to identify "hotspots" in the scheduling. Note the vertical axis on all graphs are the same.

- FindTradeofF is working with planned examination times, whereas in reality, some examinations can take longer than planned for.

The predictions are then conservative predictions of how many magnet overtime minutes occur, based on what the fairtime, $\varphi$, is desired.

\subsection{Real data}

In this section we show how our algorithms and models can be used as a decision support tool to determine time periods where potential efficiency improvements exist. We focus, as previously stated, on the scheduling for the $1.5 \mathrm{~T}$ magnet and examine which time periods contained magnet overtime for the real schedule but none or little for the algorithm. These periods are potential areas of inefficiency that can be further examined by the decision maker. By identifying these periods, the amount of data the decision maker has to consider can be reduced.

The actual overtime can be estimated from the data, by adding up the magnet times that occurred outside of the usual working day (i.e., beyond $7 \mathrm{AM}$ to $7 \mathrm{PM}$ ). We omitted weekend hours from our analysis since these appointments were never scheduled. We then ran FINDTRADEOFF on the original data set by treating each of the magnet on times as their originally scheduled times. We allowed the algorithm to reschedule their examinations within two days of their original appointment. Note that this allows the algorithm considerably more flexibility than the actual schedule. The resulting tradeoff curves are depicted in Figure 5 . Note that both the incidence and conditional overtime are shown (as described above). From the tradeoff curves, we can see that using a $\varphi$ from 0.45 to 0.55 results in an average overtime that is within the same amount of conditional overtime experienced in reality. Based on this observation, we then compared the overtime for each day from the data to overtime the algorithm attained for $\varphi=0.45$ and $\varphi=0.55$. Note that the real schedule had 94,465 minutes of magnet ontime versus the algorithm which scheduled 118, 294 minutes for $\varphi=0.45$ and 131,592 minutes for $\varphi=0.55$. These comparisons are shown in Figure 6 . As can be seen, the period from 8/20/07-11/5/07 contains days of overtime for the real schedule but none for the algorithm, even as $\varphi$ was increased.

\subsection{Simulated results}

In this section, we describe the results of our simulation when combined with FindTRADEOFF. Moreover, we describe how the results could be used to aid in determining when to purchase an additional MRI magnet. As we previously noted, we analyze data for the $1.5 \mathrm{~T}$ magnet. 
Predicted tradeoff CURVEs 2009-2018
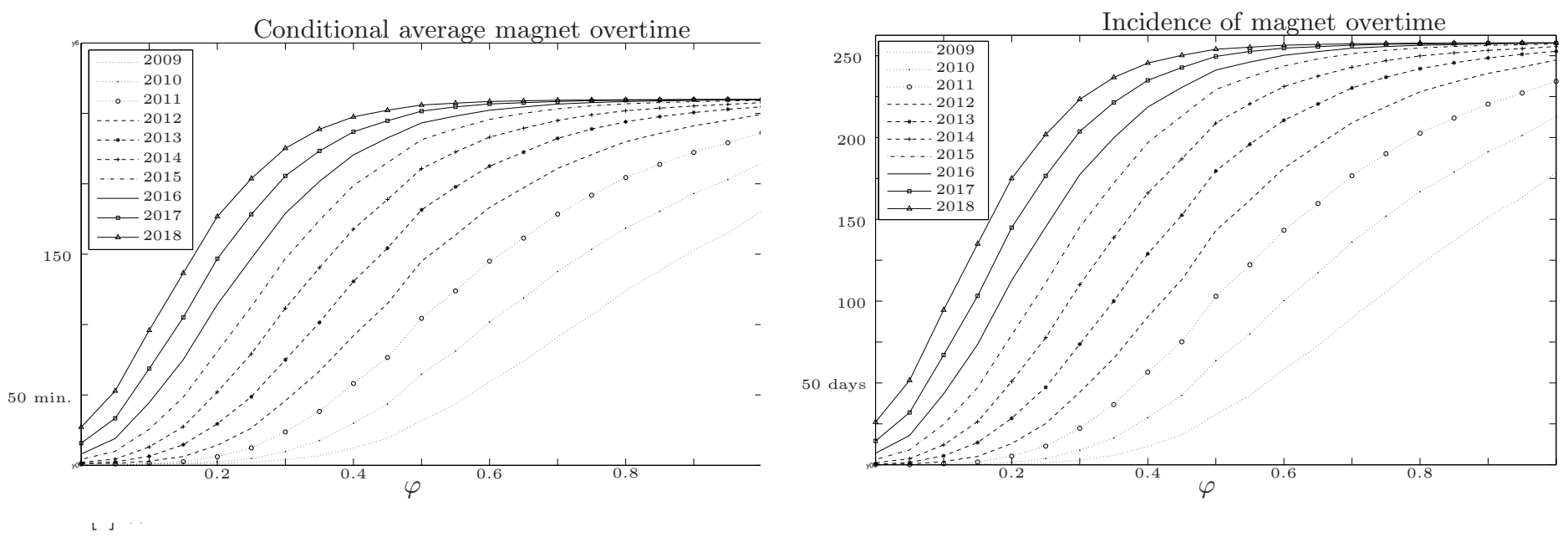

Figure 7: The two graphs show the tradeoff curves for 2009-2018 on the 1.5T magnet assuming the trends described by the 2007-2008 dataset. The graph on the left represents the average magnet overtime among days that had magnet overtime. The graph on the right represents the number of total weekdays with a magnet overtime examination. Note that the total number of weekdays in a year is 252 (excluding holidays)

We generated data for 2009 to 2018 and created schedules by using FindTradeOFF. The results of executing our algorithms are shown in Figure 7. As can be seen in the right graph, given the current trends, the predicted incidence and severity of magnet overtime steeply rises. By determining the desired $\varphi$ as described in Section 4.1, the decision maker can use the predicted results as a lower bound (see above) on the amount of magnet overtime minutes and incidence minutes. Thus, the model can predict when a new magnet would be required to avoid exceeding some cutoff for overtime incidence/minutes. For example, if $\varphi=0.3$ were desired and a threshold of 50 days of overtime were set, then under current trends, FindTRADEOFF suggests purchasing a new 1.5T magnet in 2012, as can be seen in Figure 7.

In addition to using the historical trends to generate predictions, we can also perform case analysis. We demonstrate this with two different scenarios. In the first, we simulated increased cardiac examinations by a factor of three. In the second, we decreased the overall trend by a third. Both of these cases are shown in Figure 8, along with the original simulation for comparison. As shown, the impact of increasing the cardiac examinations has a modest shift on the tradeoff curves, whereas a reduction in the overall trend results in a larger improving shift in the magnet overtime incidence. Additional graphs displaying the results of case analysis can be found in Appendix B.

\section{Discussion}

The use of technological devices has become ubiquitous in modern medical diagnosis and care. Given the cost and importance of their use, effective allocation and planning such resources is an important problem facing healthcare providers. In this paper, we have developed a tool that can perform both retrospective analysis of previous decision making as well as case analysis of the impact of possibly trends in MRI examination demand.

Response from SCH has been positive to our tool. A future study has been planned where the impact of increasing certain examination types will be analyzed using our simulation and algorithm. In addition, since the trend for MRI examinations is rising, interest has been expressed in using the tool to analyze potential impacts of purchasing an additional magnet in different years.

Our algorithm and simulation has been a positive step in developing an evaluation and prediction tool for 
PREDicted $\varphi$ Vs. INCIDENCE TRADEOFF CURVES: CASE ANALYSis
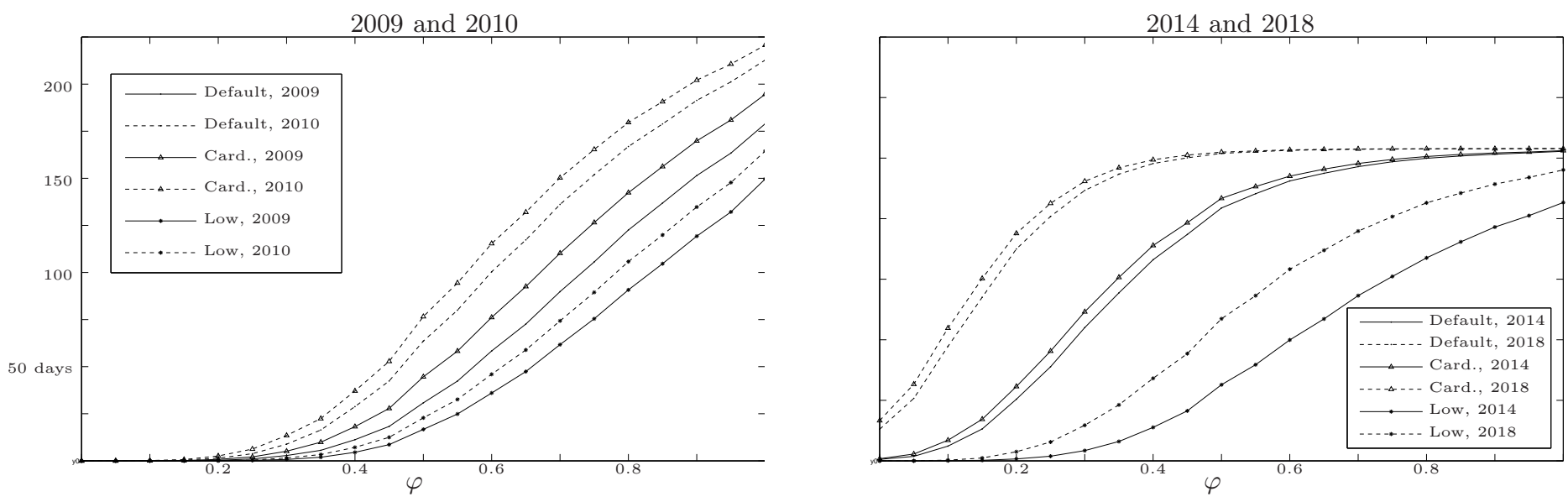

Figure 8: The two graphs show a case analysis of the predicted tradeoff curves for 2009, 2010,2014 and 2018 on the $1.5 \mathrm{~T}$ magnet between $\varphi$ and the incidence of magnet overtime days. Default is the case where the trends are as predicted by the data. Card is the case where the number of cardiac patients increases 3-fold. Low is the case where the increase in examinations predicted by the data is reduced by a third. The graph on the left depicts the predictions for 2009 and 2010. The graph on the right represents the number of total weekdays with an magnet overtime examination. Note that both graphs have the same y-axis.

better resource allocation and planning for medical technology. However, many challenges remain, including extending the tool to other treatment and diagnosis modalities such as CT-scanner scheduling. We are also interested in applying similar optimization and simulation tools to other areas where descriptive modeling is required.

\section{References}

[1] N. BAILEY, A study of queues and appointment systems in hospital out-patient departments, with special reference to waiting-times, Journal of the Royal Statistical Society. Series B (Methodological), 14 (1952), pp. $185-199$.

[2] T. Bodenheimer, High and rising health care costs. part 2: Technologic innovation., Annals of Internal Medicine, 142 (2005), pp. 932-937.

[3] T. CAyirli And E. Veral, Outpatient scheduling in health care: a review of literature, Production and Operations Management, 12 (2003), pp. 519-549.

[4] D. Cox, Regression models and life-tables, Journal of the Royal Statistical Society. Series B (Methodological), (1972), pp. 187-220.

[5] D. M. Cutler And M. MCClellan, Is technological change in medicine worth it? (cover story), Health Affairs, 20 (2001), pp. 11-29.

[6] M. Garey And D. Johnson, "Strong" NP-Completeness Results: Motivation, Examples, and Implications, Journal of the AssocmUon for Computing Machinery, 25 (1978), pp. 499-508.

[7] M. Garey And D. Johnson, Computers and Intractability: A Guide to the Theory of NPCompleteness, W.H. Freeman and Company, New York, 1979. 
[8] L. Green, S. Savin, And B. Wang, Managing patient service in a diagnostic medical facility, Operations Research, 54 (2006), pp. 11-25.

[9] D. Gupta And B. Denton, Appointment scheduling in health care: Challenges and opportunities, IIE Transactions, 40 (2008), pp. 800-819.

[10] B. Kalyanasundaram and K. Pruhs, On-line weighted matching, in Proceedings of the 2nd ACMSIAM Symposium on Discrete Algorithms, 1991, pp. 234-241.

[11] R. Kolisch And S. Sickinger, Providing radiology health care services to stochastic demand of different customer classes, OR Spectrum, 30 (2008), pp. 375-395.

[12] L. Leemis And S. Park, Discrete Event Simulation, A First Course, Prentice-Hall, Inc., Upper Saddle River, NJ, 2006.

[13] J. Patrick And M. L. Puterman, Improving resource utilization for diagnostic services through flexible inpatient scheduling: A method for improving resource utilization, Journal of Operational Research Society, 58 (2008), pp. 235-245.

[14] D. B. Shmoys And E. TARdos, An approximation algorithm for the generalized assignment problem, Mathematical Programming A, 62 (1993), pp. 461-474.

[15] S. Sickinger And R. Kolisch, The performance of a generalized Bailey-Welch rule for outpatient appointment scheduling under inpatient and emergency demand, Health care management science, 12 (2009), pp. 408-419.

[16] I. Vermeulen, S. Bohte, S. Elkhuizen, H. Lameris, P. Bakker, and H. Poutré, Adaptive resource allocation for efficient patient scheduling, Artificial intelligence in medicine, 46 (2009), pp. 6780.

[17] J. Welch And N. T. J. BAILey, Appointment systems in hospital outpatient departments, The Lancet, 259 (1952), pp. 1105-1108. 


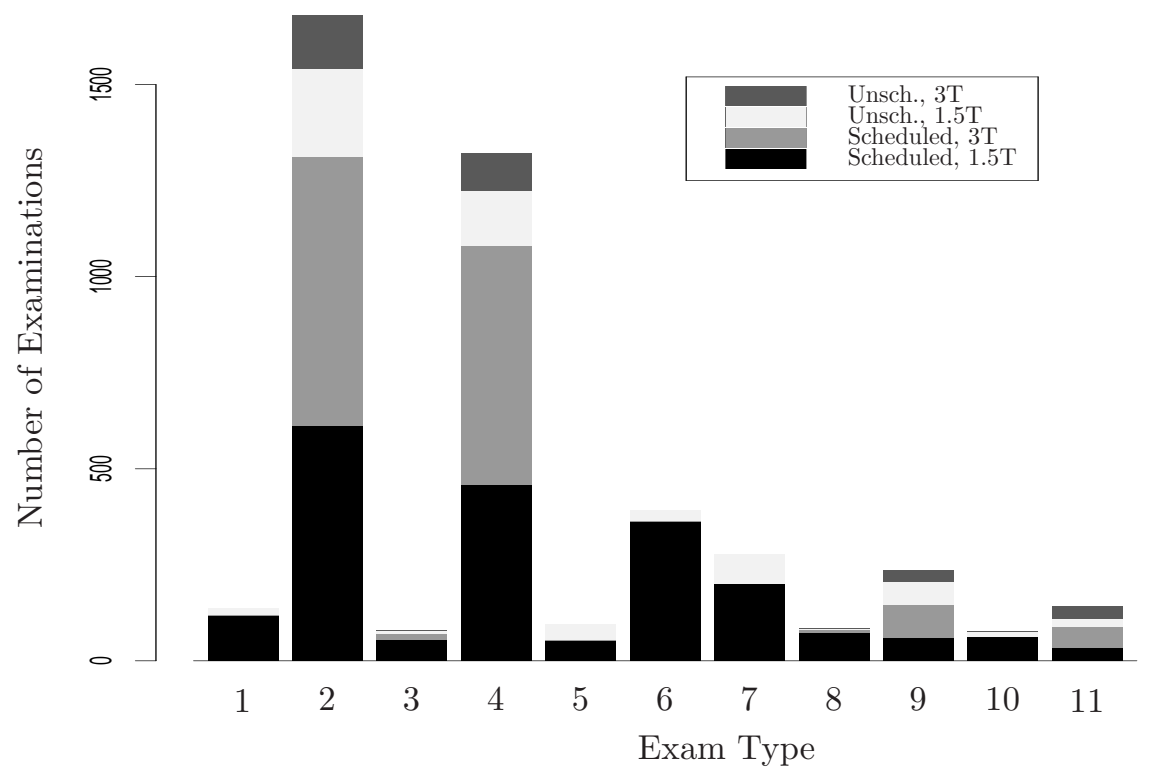

Figure 9: Number of scheduled and unscheduled examinations on 1.5T and 3T magnets. Each bar represents the total number of examinations for a single examination type. The shaded areas within each bar represent the number of examinations in each of four different categories (e.g. scheduled, 1.5T examinations). All examination types with at least 50 examinations in one of the four categories are shown here.

\begin{tabular}{clcl}
\hline Exam & Examination Type & Exam & Examination Type \\
\hline 1 & Spine lumbar without contrast & 2 & Brain with and without contrast \\
3 & Abdomen with and without contrast & 4 & Brain without contrast \\
5 & Spine total without contrast & 6 & Spine total without contrast \\
7 & Spine total with and without contrast & 8 & Face and neck with and without contrast \\
9 & Angio head without contrast & 10 & Spine cervical without contrast \\
11 & Brain limited & & \\
\hline
\end{tabular}

\section{A Further details of the data}

In this appendix, we describe some of the further details of our data.

In Figure 9 it can be seen that no magnet preference was exhibited by unscheduled arrivals.

\section{A.1 Datafield descriptions}

We describe our data fields in Table 2. In order to mask the medical record number (see the datafield deidentified.MRN), a new number was generated to be used instead of the original MRN as specified in the internal review boards guidelines.

\section{A.2 Omitted data}

We omitted 17 data records for reasons detailed in Table 3. When multiple reasons existed for omitting a record, we describe the most significant.

In addition to the data records removed as stated above, we also removed the following weekdays from our trend data set. Days removed due to maintenance/training were 02/04/03, 02/20/03, 02/21/03, 06/03/03, 08/05/03, 10/07/03, 11/20/03, 01/06/04, 01/07/04, 04/01/04, 07/26/04, 09/16/04, 05/24/05, 05/26/05, 


\begin{tabular}{l|l} 
Datafield name & Description \\
\hline row.names & Internal ID \\
procedified.MRN & masked medical record number \\
gender & gender \\
date.of.exam & date of exam (MM/DD/YY) \\
years & age in years \\
months & age in months \\
days & age in days \\
start.time.of.exam & time magnet activated \\
end.time.of.exam & time magnet deactivated \\
time.of.attending.signoff & time attending MD signed report \\
date.of.attending.signoff & date attending MD signed report \\
time.of.resident.dictation & time resident dictated report \\
date.of.resident.dicatation & date resident dictated report \\
outpatient & whether outpatient or not \\
scheduled & whether scheduled or not \\
scheduled.time & scheduled time, if applicable \\
1.5.tesla & whether 1.5 T magnet used \\
contrast & whether contrast used \\
repeat.exam & whether examination was repeated \\
diagnosis & diagnostic code \\
total.aquired.sequences & total sequences taken \\
anasthesia & indicates whether anasthesia used \\
status & patient status \\
cancelled & indicates whether patient cancelled \\
\hline
\end{tabular}

Table 2: Datafield descriptions

10/14/05, 10/21/05, 10/25/05, 10/26/05, 10/27/05, 10/28/05, 11/07/05, 11/09/05, 11/10/05, 11/11/05, 12/08/05, 02/01/06, and 06/02/08. Days removed for holidays each year were Martin Luther King Day, President's Day, Independence Day, Labor Day, Memorial Day, Thanksgiving, Christmas Day, and New Year's Day.

\section{B Further simulated data}

Here, we display additional case analyses of tradeoff and overtime curves that result from the cases of (1) increasing the number of cardiac patients (Figure 10); and (2) scaling the increasing trend of MRI examinations by a third (Figure 11). 
Examination type

Functional Brain Study w/ MD or Psych

Functional Brain Study w/ MD or Psych

Angio Head without Contrast

Cardiac without Contrast

Brain without Contrast

Brain with \& without Contrast

Angio Head without Contrast

IAC without contrast

Spine Total with \& without Contrast

Ext Upper Rt with \& without Contrast

Brain Limited

Abdomen with \& without Contrast

Joint Lower Rt wout Contrast

Joint Lower Rt with \& without Contrast

Right Ankle without Contrast

Brain with \& without Contrast

Spine Cervical without Contrast
Reason for omission

missing 19 data fields

missing 19 data fields

missing status

missing start and end times

missing age

examination time exceeding 1300 minutes

examination time exceeding 1400 minutes

examination time exceeding 800 minutes

examination time exceeding 1400 minutes

examination time exceeding 1300 minutes examination time exceeding 1300 minutes

Unknown magnet

Unknown magnet

Unknown magnet

Unknown magnet

Age over 18

Age over 18

Table 3: Omitted examination records

\section{Simulated TRADEOFF CURVES 2009-2018, INCREASED CARDiAC PATIENTS}
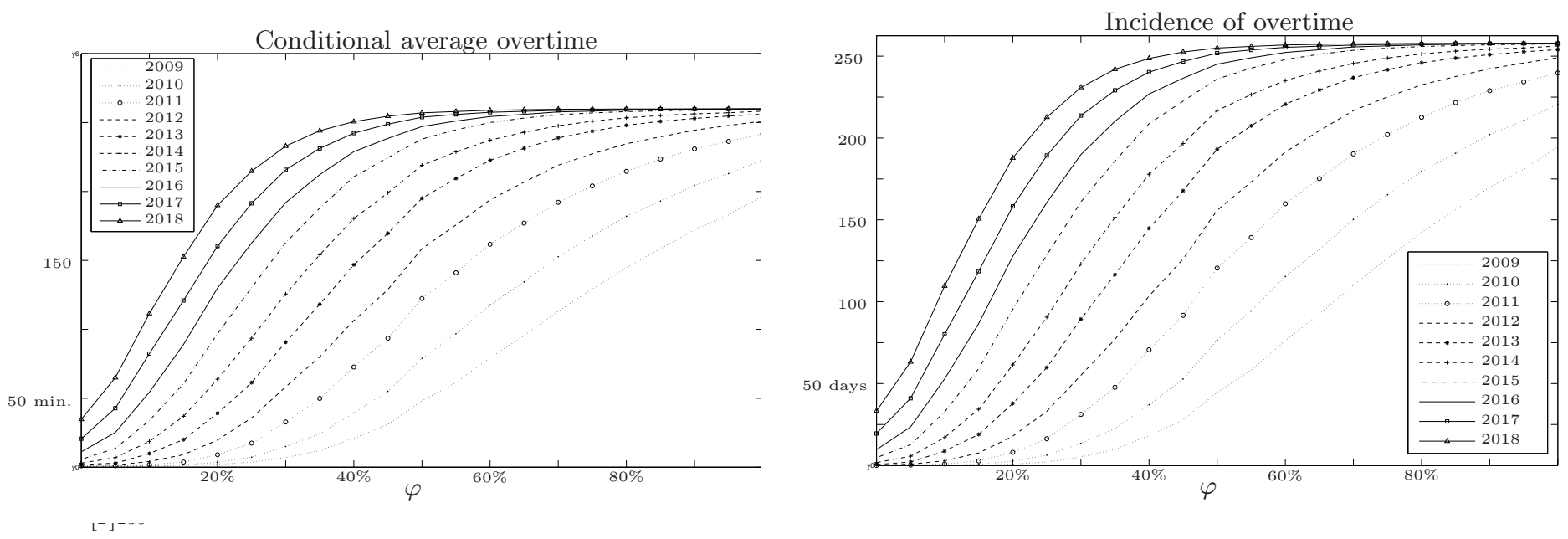

Figure 10: The two graphs show the tradeoff curves for 2009-2018 on the 1.5T magnet assuming the trends described by the 2007-2008 dataset with the cardiac examinations tripled. The figure on the left represents the average overtime figure among days that had overtime. The figure on the right represents the number of total weekdays with an overtime examination. Note that the total number of weekdays in a year is 252 (excluding holidays) 


\section{Simulated TRAdeoff CURVEs 2009-2018, TREnd Divided By THREE}
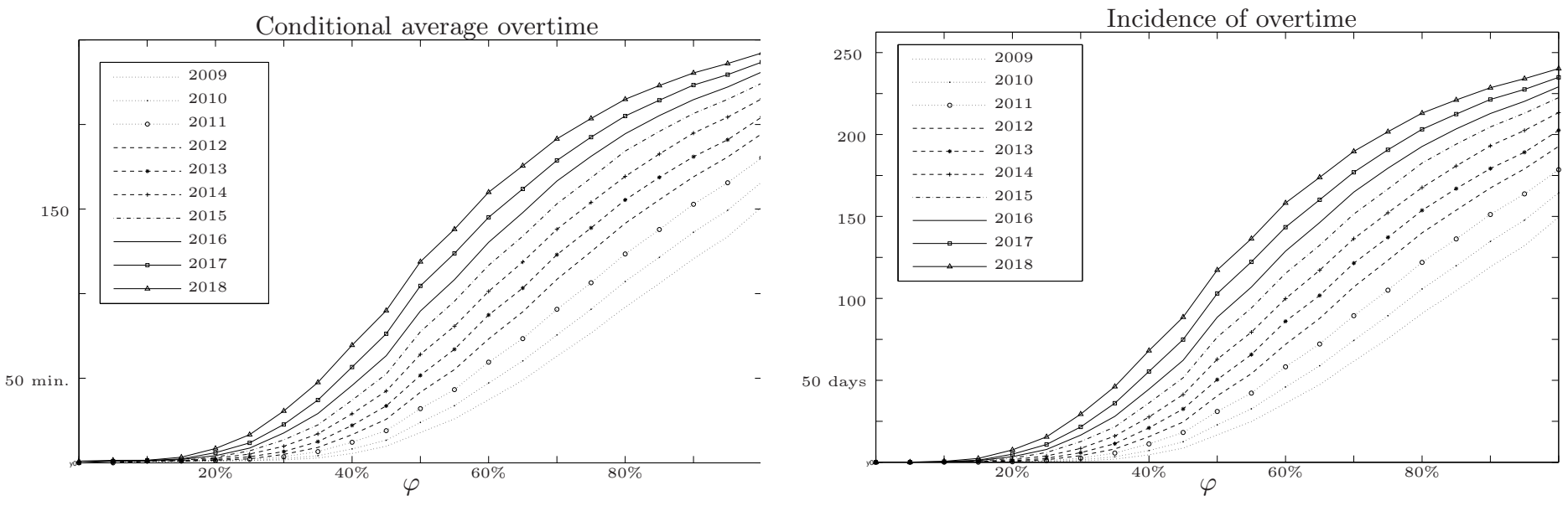

Figure 11: The two graphs show the tradeoff curves for 2009-2018 on the 1.5T magnet assuming only one third of the trends described by the 2007-2008 dataset. The figure on the left represents the average overtime figure among days that had overtime. The figure on the right represents the number of total weekdays with an overtime examination. Note that the total number of weekdays in a year is 252 (excluding holidays) 